\title{
BMJ Open Computerised cognitive behaviour therapy for depression in adolescents: study protocol for a feasibility randomised controlled trial
}

Barry Wright, ${ }^{1}$ Lucy Tindall, ${ }^{1}$ Elizabeth Littlewood, ${ }^{2}$ Joy Adamson, ${ }^{2}$ Victoria Allgar, ${ }^{2}$ Sophie Bennett, ${ }^{1}$ Simon Gilbody, ${ }^{2}$ Chrissie Verduyn, ${ }^{3}$ Ben Alderson-Day, ${ }^{1}$ Lisa Dyson, ${ }^{2}$ Dominic Trépel, ${ }^{2}$ Shehzad $\mathrm{Ali}^{2}$

To cite: Wright $B$, Tindall $L$, Littlewood E, et al. Computerised cognitive behaviour therapy for depression in adolescents: study protocol for a feasibility randomised controlled trial. BMJ Open 2014:4:e006488. doi:10.1136/bmjopen-2014006488

- Prepublication history and additional material is available. To view please visit the journal (http://dx.doi.org/ 10.1136/bmjopen-2014006488).

Received 28 August 2014 Accepted 11 September 2014

\section{CrossMark}

${ }^{1}$ Limetrees Child Adolescent and Family Unit, York, UK ${ }^{2}$ The University of York, York, UK

${ }^{3}$ Manchester Children's Hospital NHS Trust, Manchester, UK

Correspondence to Professor Barry Wright; barry.wright1@nhs.net

\section{ABSTRACT}

Introduction: The 1 year prevalence of depression in adolescents is about $2 \%$. Treatment with antidepressant medication is not recommended for initial treatment in young people due to concerns over high side effects, poor efficacy and addictive potential. Evidence suggests that cognitive behaviour therapy (CBT) is an effective treatment for depression and is currently one of the main treatment options recommended in adolescents. Given the affinity young people have with information technology they may be treated effectively, more widely and earlier in their illness evolution using computeradministered CBT (CCBT). Currently little is known about the clinical and resource implications of implementing CCBT within the National Health Service for adolescents with low mood/depression. We aim to establish the feasibility of running a fully powered randomised controlled trial (RCT).

Methods and analysis: Adolescents aged 12-18 with low mood/depression, (scoring $\geq 20$ on the Mood and Feelings Questionnaire (MFQ)), will be approached to participate. Consenting participants will be randomised to either a CCBT programme (Stressbusters) or accessing selected websites providing information about low mood/depression. The primary outcome measure will be the Beck Depression Inventory (BDI). Participants will also complete generic health measures (EQ5D-Y, HUI2) and resource use questionnaires to examine the feasibility of cost-effectiveness analysis. Questionnaires will be completed at baseline, 4 and 12month follow-ups. Progress and risk will be monitored via the MFQ administered at each treatment session. The acceptability of a CCBT programme to adolescents; and the willingness of clinicians to recruit participants and of participants to be randomised, recruitment rates, attrition rates and questionnaire completion rates will be collected for feasibility analysis. We will estimate

'numbers needed' to plan a fully powered RCT of clinical and cost-effectiveness.

Ethics and dissemination: The current trial protocol received a favourable ethical opinion from Leeds (West) Research and Ethics Committee. (Reference: 10/H1307/137).

Trial registration number: ISRCTN31219579.

\section{Strengths and limitations of this study}

The study addresses an under-researched area and will produce important feasibility information to inform a fully powered randomised controlled trial (RCT).

- The study explores the feasibility of using a range of mental health and health economics measures.

- Feasibility data on acceptability, completion rates and attrition are supplemented by participant qualitative interviews.

- The study explores the feasibility of various economic analysis measures in a population of adolescents with low mood.

- The sample of participants is obtained from only one NHS Trust resulting in potential bias. A future fully powered RCT will be extended to other localities.

\section{INTRODUCTION}

Tricyclic antidepressants have no benefit over placebos in the treatment of adolescent depression $^{1}$ and are not recommended for treatment in this group by the National Institute for Health and Care Excellence (NICE) guidelines. ${ }^{2}$ At the time of ethical approval for this study, the European Medicines Agency $^{3}{ }^{4}$ recommended that SSRIs in general should not be used for the treatment of depression in young people, with the exception of fluoxetine where there was some evidence, although limited, of its efficacy. There are additional concerns about the addictive potential of antidepressants and many young people and parents would prefer a 'talking' therapy in the first instance. $^{5} \quad 6$ The argument against using medication early is further strengthened by the finding of a large study showing that $21 \%$ of moderate to severely depressed adolescents improved with a brief psychosocial 
intervention before being randomised between medication and treatment as usual. ${ }^{7}$ There is thus a climate of caution about antidepressant use in general and particularly in adolescents, due to evidence of poor efficacy and high side effects. Research therefore needs to focus on investigating the use of more acceptable and accessible psychosocial treatments for adolescent depression.

Reviews of cognitive behavioural therapy (CBT) for adolescent depression have shown that it is effective and currently one of the main treatment options recommended in adolescents. ${ }^{2}{ }^{8}$ Trials comparing CBT to either family therapy or supportive psychotherapy show that it is better at both improving mood and achieving remission. ${ }^{9}$ It has also been shown to prevent depression in high-risk adolescent groups including the offspring of depressed adults. ${ }^{10}$ Since it has a low side effect profile it is an attractive alternative option for the treatment of adolescent depression. However, some studies have questioned its efficacy in young people with moderate to severe depression, questioning how powerful a treatment it is. ${ }^{711}$

The 1 year prevalence of depression in adolescents is about $2 \% .{ }^{12}$ An area with a population of 300000 , such as that served by the York and Selby Community Adolescent Mental Health Service (CAMHS), would be expected to have at least 450 young people with depression. Delivering CBT to this number of young people is far more resource intensive in terms of time than using medication. Many primary care doctors are referring all young people directly in to CAMHS rather than managing them in primary care, as they are reluctant to use medication and do not have the time or experience to deliver psychological therapy. Most primary care counsellors are not trained to counsel adolescents. This creates an additional pressure in CAMHS. Together with other service pressures, this leads to long waiting lists in CAMH services in some areas. As a result, it is unlikely that individual CBT needs can be met from existing therapist resources. ${ }^{13}$ This therefore presents a number of challenges to services, including demands on clinical time, the potential development of waiting lists and issues about prioritisation of services.

Given the affinity young people have with information technology, it may be that young people could be treated effectively and more widely using CCBT and at an earlier stage in the evolution of their illness. The Improving Access to Psychological Therapies (IAPT) programme was introduced to improve availability of therapies and specifically mentions the use of CCBT in relation to depression. CCBT represents an alternative form of therapy delivery, which has the potential to enhance access to CBT and to provide a realistic alternative therapy or potentially a preventive intervention early in the course of depressive illness. Some argue that many adolescents, often reluctant to engage one to one with a therapist, may be more comfortable accessing computerised material or therapy.

CCBT comes in many forms and can be directed at a range of conditions and age groups, but adequate research needs to be conducted to capture this broad range of possible new therapies. Existing research into CCBT, which has mainly been conducted with adults with depression, has most recently been summarised in a 2006 HTA review of clinical and cost-effectiveness. ${ }^{14}$ With respect to depression, three commercially-produced computerised packages available to the National Health Service (NHS) were considered-Beating The Blues, Cope and Overcoming Depression. Of these, only one adult intervention, Beating The Blues, best suited to those aged 25 and over, had been evaluated in a randomised controlled trial by its developers. ${ }^{13}$ An internet package, MoodGYM, used with adults has been evaluated in a general adolescent population but $70 \%$ of young people did not complete the programme. ${ }^{15}$

The CCBT package Stressbusters, (developed by a team from Manchester, The Institute of Psychiatry, London and Australia), combining expertise in CBT and computer-based delivery was specifically developed for use in an adolescent population. The programme has had a very encouraging case series where the programme was successfully used with over 30 adolescents, $70 \%$ of whom completed the full eight sessions. ${ }^{16}$ At initial assessment, $95 \%$ of the sample met diagnostic criteria for a depressive disorder, with a high group mean score (34.48, SD 9.84) on the Moods and Feelings Questionnaire (MFQ). Post-treatment, this figure had fallen to $22 \%$ with the group mean score on the MFQ falling significantly below clinical cut-off (20.32, SD 11.75). These gains were maintained at 3-month follow-up. While computer-based approaches have demonstrated positive results for adolescents with anxiety $\left(\mathrm{eg}\right.$, Coolteens ${ }^{17}$; BRAVE ${ }^{18}$ ), there are as yet no trials of computerised CBT for adolescents with depression.

The use of CCBT to treat depression is therefore a potentially effective and efficient way of enhancing access to psychological therapies in an adolescent population. However, this is an unevaluated technology. Since there is a paucity of published research evidence on the use of CCBT for adolescent depression, a feasibility study is required to establish: the willingness of clinicians to recruit participants and the willingness of participants to be randomised; the acceptability and utility of a range of outcome measures; recruitment rates; attrition rates and an estimation of sample size needed for a fully powered RCT. We also explored the acceptability of CCBT to adolescents, including their satisfaction and compliance with the programme and venue.

This feasibility study is necessary preparatory work for a fully powered definitive RCT and will produce important research evidence to inform the care of young people in the UK NHS.

\section{METHODS AND ANALYSIS}

\section{Participants and recruitment}

Our target population is 12-18-year-olds with low mood. Currently in our local CAMHS patch (York and Selby) 
all referrals go through a sectorised primary mental health worker (PMHW) system, with 8 PMHWs covering 2-3 secondary schools, all the feeder primary schools and local General Practitioner (GP) surgeries. Referrals can be made by any professional in contact with children and young people (eg, teacher, GP, paediatrician, social worker, etc). We also take referrals from a young persons drop in centre run by the local authority, situated in the centre of York, often accessed by young people who are out of school. Currently therefore, young people out of school with low mood are referred by these professionals. While we cannot guarantee that all such young people reach our service, it is set up to make it as accessible as possible. This provides a unique opportunity to monitor and assess any young person in our geographical area with low mood or depression.

The PMHWs will assess all young people who present with any mood disorder referred through this system. Any young person with a mood disorder at assessment would usually be referred to tier 2, 3 or 4 CAMHS by the PMHWs. During the study, young people who score 20 or above on the MFQ (a validated screening tool for this age group used in many research studies) will be referred to the trial by the PMHWs. They will be given an information leaflet with a copy for their parent/guardian and offered a place on the trial. Full informed consent will be obtained from young people and, where a young person is under 16 years, their parent/guardian.

Even with this comprehensive referral system, we have in place plans to conduct a second recruitment method within schools if we fail to meet our recruitment targets using the above outlined approach. Here, with informed consent from governing bodies/head teachers and local authority approval, we will recruit from local schools. In this way the study recruitment will rotate through the target schools. Children in year 7 to Upper Sixth between the ages of 12-18 in local secondary schools will be screened using the MFQ and recruited systematically.

The parents of all young people in year 7 to Upper Sixth in all recruiting schools will receive an information leaflet explaining the study to them. Participation is entirely voluntary, children and their families wishing to take part in the study will opt-in using the consent form and the stamped addressed envelope provided.

\section{Inclusion criteria}

Our target population will be adolescents aged 12-18 with low mood/depression. Our inclusion threshold will be a MFQ score of 20 or above, which has $70 \%$ sensitivity and $81 \%$ specificity for any mood disorder. ${ }^{19}$ The cut-off for a major depressive episode is 29. We will also include participants with either comorbid physical illness or comorbid non-psychotic functional disorders, such as anxiety.

\section{Exclusion criteria}

We will exclude participants who are seeking to end their life, suffering psychotic symptoms or depressed in the postnatal period. Participants with previous depression or previous treatment with antidepressants or experience of cognitive therapy will not be excluded. We will exclude cases of psychotic depression, since computerised therapy for this group is not recommended within NICE guidance. ${ }^{20}$

\section{Study design}

The study will be conducted between June 2011 and December 2014. We will use a RCT methodology with remote randomisation (provided by York Clinical Trials Unit at the University of York). Participants who meet our pragmatic inclusion criteria and who score 20 or above on the MFQ will be consented into the trial and will be asked to provide demographic information and complete:

1. The short Beck Depression Inventory (BDI)

2. The MFQ

3. The Spence Children's Anxiety Scale (SCAS)

4. A risk factor schedule

5. A Quality of Life and Resource Use questionnaire

Consented participants will then be randomised to either Arm 1 or Arm 2.

\section{Intervention}

Arm 1: CCBT using the program 'Stressbusters'

Stressbusters is a CCBT package developed by a team from Manchester, The Institute of Psychiatry, London and Australia (combining expertise in CBT and computerbased delivery).

The Stressbusters software application consists of eight 30-45 min sessions of CCBT designed for 12-18 year olds. The programme is based on the manualised treatment programme from an RCT designed to evaluate effectiveness of CBT compared to a placebo control. ${ }^{21}$ Each Stressbusters session is an interactive presentation featuring narration synchronised with videos, animations, graphics and printouts.

The programme has a narrator guiding participants through eight sessions in linear progression, with each building on the knowledge gained in previous sessions and on the tasks carried out at home. A session or part of a session can be repeated wherever necessary. Sessions contain flexible 'add-ons' such as written fact sheets (eg, about bullying, sleep problems) which can be printed out and taken away, together with home practice related handouts from the programme (eg, mood diary sheets).

The video inserts (case vignettes) are of three teenagers who feature throughout. They are student actors who play the roles of depressed teenagers, specially scripted and created for the project. Participants hear about the lives of the teenagers and watch them using the programme in a combination of short video sequences and voiceovers.

The session content is organised into the following format: 
Session 1: Introduction to the programme and goal setting

Session 2: Getting activated

Session 3: Emotional recognition

Session 4: Noticing thoughts

Session 5: Thought challenging

Session 6: Problem solving

Session 7: Improving social skills

Session 8: Relapse prevention

\section{Arm 2: websites}

This involves equivalent time to access selected websites chosen by an expert clinical panel containing general safe and official information on low mood/depression and self-help. The selected websites are:

http://www.youngminds.org.uk

http://www.RU-OK.org.uk

http://www.youthhealthtalk.org

http://www.depressioninteenagers.com/

All participants will also be asked to complete the MFQ and BDI questionnaires at 4 months and 1 year after completion of/withdrawal from trial sessions. We will test the feasibility of using a likert scale to explore treatment preference as this could be used as a measure in a fully powered trial.

Outside the trial most young people referred with low mood/depression would usually be offered appropriate treatment based on their symptomatology and severity. This would most likely include face-to-face CBT, family therapy, medication and in some instances admission to hospital. This is a pragmatic trial and no participants will be denied access to additional treatments and services (offered under routine NHS care) by virtue of participating in this trial. Both arms in the trial will also receive care as usual and this could include any of the following. These will be monitored and recorded for each participant:

- Local counselling services (eg, youth enquiry and support services; tier 1).

- Primary mental health worker support (tier 1).

- Individual supportive therapy or brief psychosocial interventions (tier 2).

- Individual CBT (tier 2).

- Psychiatric referral (tier 2).

- Family therapy (tier 3).

- Group work for self-harm or depression (tier 3).

- In-patient admission (tier 4).

- Medication (while medication is not currently recommended in this group, we will monitor whether any professional prescribes).

\section{Procedure}

Participants in the CCBT group will complete eight sessions of the Stressbusters programme lasting approximately $45 \mathrm{~min}$ (with homework between sessions) at a private and user-friendly location. Participants using selfhelp websites on low mood will be offered equivalent time to access the websites in the same settings.
Participants will be given a choice of setting to participate including CAMH clinic sites, schools, a GP surgery or a local community centre. A member of the research team will meet all participants at each of their sessions to provide them with instructions. During the sessions the researcher will wait outside of the room where the session takes place but will remain in close proximity to assist participants if they have any difficulties and answer any questions.

It will be explained to all participants that care as usual will be available to them. For example each school and primary care practice will have a primary mental health worker (PMHW) who will liaise to ensure that any professional who has concerns about any young person can discuss their concerns and make a referral if necessary. PMHWs can provide up to four sessions of support or refer individuals on to an accessible member of the specialist CAMHS team if necessary. They will also provide consultation to other professionals supporting the young people as usual where necessary (eg, school nurses, school counsellors or mentors, school tutors, Young People Advisory Service workers and other relevant professionals). PMHWs will be able to access tier 2 and tier 3 professionals at any time urgently or nonurgently in the usual way by direct referral through a central allocation system weekly or through a daily 'duty clinician' system or an urgent on call psychiatry system, as appropriate. In this way they can be allocated to a CAMHS professional for treatment in the usual way either routinely or urgently at any time.

Standardised instruments will be used to trawl hospital and mental health notes to record episodes of contact or treatment with any CAMHS professional. The unified NHS appointment and care tracking system will also be used.

(The trial process is diagrammatically presented in online supplementary appendix 1 ).

\section{Outcome measures}

We will examine whether CCBT affects outcome in terms of:

Feasibility outcome measures

- The acceptability of a CCBT programme for adolescents.

- The willingness of clinicians to recruit and young people to be randomised.

- Numbers of eligible participants and recruitment rates.

- Adherence to treatment and outcome measures.

- Time needed to collect data.

- The SDs of outcome measures to estimate sample size in a fully powered RCT.

Clinical outcome measures

- Scores on the short BDI questionnaire (primary outcome measure).

- Scores on the MFQ the Spence Anxiety Scale, the EQ5D-Y, the HUI2 (Health Utilities Index) and the resource use questions. (Measures of health-related 
quality of life and resource use provide the bases to evaluate whether cost-effectiveness would be feasible as part of a fully powered study).

- Information relating to progression to further treatment, episodes of self-harm and any inpatient admissions.

\section{Qualitative outcomes}

Acceptability of the intervention and the trial process will also be studied using a qualitative approach.

We will conduct qualitative interviews with a purposively sampled group of study participants. Based on previous research, a sample of 20 participants should be sufficient to collect adequate data. ${ }^{22}$ The purposive sampling frame will ensure maximum variation within the qualitative sample on the basis of age, gender and depression score. Most (15) participants will be from the CCBT arm of the trial. A smaller number (5) of participants will be included from both withdrawals from treatment and the websites group. All 20 participants will be interviewed once after randomisation but prior to disclosure of allocation and again 1 week after their completion/withdrawal from the CCBT programme (or equivalent time for those accessing self-help websites). Qualitative interviews will be conducted using a topic guide to ensure consistency across participants.

During the qualitative interviews, information will be collected on topics including experiences of depression, responses to symptoms of depression and which health outcomes would be of the greatest value to this particular group. Data will also be collected on the trial process itself and include questions on the acceptability of the treatment and location, the randomisation procedure and methods of data collection.

Qualitative interviews will be audio recorded digitally and transcribed verbatim. Data will be managed in Atlas/ ti or NVivo 9 and will be analysed according to the constant comparison method through thematic coding of the data. ${ }^{23}$ The coding will be a combination of a priori themes-largely relating to the acceptability of the treatment and the trial process evaluation, alongside emerging themes from a more in-depth analysis drawing on theoretical models of illness behaviour in the context of mental illness including Pescosolido's network-episode model $^{24} 25$ and Biddle's cycle of avoidance. ${ }^{26}$ The primary output from the qualitative work will be data on the acceptability of both the trial intervention and the trial process, which will inform the design of a full-scale trial. The qualitative interviews conducted 1 week postintervention will explore participants' views about the programme, their satisfaction, and perceived usefulness of the programme, their views about venue of delivery and their experience of trial participation.

\section{Proposed sample size}

No formal power calculations are undertaken in feasibility studies; rather sufficient participants are recruited to determine factors such as attrition and recruitment rates in relation to feasibility outcomes. ${ }^{27}$ Thus we have based our sample size calculations on case study work using the Stressbusters CCBT programme in 23 young people. ${ }^{16}$ To detect a difference of 10 points posttreatment on the MFQ between the two groups, $80 \%$ power and 5\% significance, 26 participants per group are required. In a previous sequential case series only 15 participants out of 28 identified (54\%) completed all eight sessions of CCBT. ${ }^{16}$ Therefore, we will need to recruit 48 per group to account for attrition. A literature review examining CBT for anxiety and depressive disorders in children and adolescents ${ }^{8}$ found that 9 of 12 substantive studies reviewed had between 15-30 recruits in each arm. We will therefore seek to recruit four young people every month, over a 24-month period. Eight PMHWs will be recruiting participants therefore each will seek to recruit one participant every 2 months. Given that the only previous study ${ }^{16}$ of this intervention was a case series and not a RCT, the current study will provide feasibility data for a definitive trial.

\section{Data analysis}

In line with recommendations about good practice in the analysis of feasibility studies, ${ }^{28}$ analysis will be descriptive and no statistical comparisons of the outcomes between the two arms of the trial will be conducted.

Descriptive statistics will be calculated for recruitment rates, follow-up rates, attrition and adherence. Adherence to treatment calculated will be the percentage in the Stressbusters group completing all eight sessions, and of the website group completing all four websites and at least four sessions.

Descriptive data will be presented for the baseline characteristics as means and SDs or 95\% CIs, medians and IQR or percentages. Descriptive statistics will also be calculated for the outcome measures (BDI, MFQ and SCAS) at baseline, 4 months and 12 months follow-up, and the change in scores from baseline. Means and SDs, medians and IQRs will be presented. The data will be used to develop estimates for a fully powered RCT. All analyses will be undertaken on SPSS (V.21).

\section{Trial status}

Recruitment of participants is completed. The first participant was enrolled in October 2011. The last participant will complete follow-up in December 2014.

\section{ETHICS AND DISSEMINATION \\ Ethical and safety considerations}

People with low mood/depression are vulnerable. However, we do not anticipate any major ethical issues with the proposed study. All adolescents will be able to access treatment as usual and will be informed of this. The study interventions will be in addition to this. We do not anticipate any risks to young people through participation. All participants will be able to access an emergency duty clinician both within hours and on call and 
can access additional or alternative treatments. There are local arrangements for urgent contact with PMHWs between 9:00 and 17:00 on weekdays. In addition there are CAMHS duty clinicians on a rota for urgent assessments during the same hours. There is also a $24 \mathrm{~h} 7$-day per week on call service provided by a rota of consultant psychiatrists.

\section{Informing potential trial participants of possible benefits and known risks}

The participant information leaflets will provide potential participants with information about the possible benefits and known risks of taking part in the trial. Participants will be given the opportunity to discuss this with either their PMHW or research coordinator prior to consenting to participate. The research coordinator will inform the participant if new information comes to light that may affect their willingness to participate in the trial.

Regarding benefits, each participant will be given a $£ 10$ gift voucher following return of their 4-month follow-up questionnaires as a token of gratitude for their participation.

\section{Obtaining informed consent from participants}

Potential participants will receive an information leaflet about the trial and one for their parent/guardian (if under 16 years). The information leaflets will be produced using the current guidelines for researchers on writing information sheets and consent forms. Participants who wish to participate in the trial will sign a consent form along with that of their parent/guardian (if under 16 years). The researchers will then arrange a baseline visit with them to discuss the trial, answer any questions they may have and complete all baseline measures. Only after written consent and the completion of baseline measures will randomisation occur.

\section{Data protection/confidentiality}

All participant information will be stored in accordance with the Data Protection Act 1998. Participant personal identifiable information will be stored in a locked filing cabinet. All participant data will be anonymised by allocating each participant with an ID number. Anonymised participant data will be saved on a password-protected secure computer drive which only members of the research team will have access to. Participant personal identifiable data will be stored in a separate location to anonymised participant data. All data will be maintained by the research coordinator.

Laptops will be purchased to enable delivery of the CCBT and self-help websites. These laptops will be encrypted by either IT Services within Leeds and York partnership NHS Foundation Trust, or by purchasing the software package Smoothwall (as recommended by the trust), at an additional cost. This encryption will ensure that data, including personal identifiable data, can be stored safely on all laptops. In addition, encrypted memory sticks will be used to transfer data from the laptops used for intervention delivery and the computer used by the research coordinator. All personal data will be destroyed 6 months after the study ends and all study data will be destroyed after 10 years.

\section{Research governance and the conduct of the trial}

The trial will be conducted to protect the human rights and dignity of the participant as reflected in the 1996 version of the Helsinki Declaration. Participants will not receive any financial inducement to participate. In order to protect the trial participants the following will apply:

- the trial has been designed to minimise pain, discomfort and fear;

- the trial has been designed to minimise any foreseeable risk in relation to the treatments involved;

- the explicit wishes of the participants will be respected including the right to withdraw from the trial at any time;

- the interest of the patient will prevail over those of science and society;

- provision will be made for indemnity by the investigator and sponsor;

- contact details for further information will be provided.

\section{Dissemination of research findings}

We recognise that successful dissemination requires a preplanned strategy that considers the groups who need to be aware of the results of the review and the methods with which to communicate with these groups. Therefore, we will begin to consider our dissemination strategy at an early stage of the project and consult patient and public involvement (PPI) representatives throughout all dissemination processes. Our PPI representatives will particularly be consulted in relation to characteristics of the audience to be targeted, appropriate communication channels and the wider working environment of our audiences.

Throughout the trial, newsletters will be produced and forwarded to professionals within our CAMH service to promote the trial and provide updates on its progress. Furthermore all study findings will be disseminated through various channels. The main outcomes and study findings of this research will be disseminated via publication in a range of peer-reviewed journals. Presentations of study findings will also be taken to relevant research conferences, local research symposiums and seminars for CAMHS professionals, while a short summary of the results will be distributed to all trial participants. Other professionals including GPs and teachers will also be informed of study findings and their implications for patient care.

\section{Clinical trials authorisation and ethical approval}

No new pharmaceutical compounds are used in this trial and clinical trials authorisation is not required. 


\section{Trial sponsorship}

The trial is sponsored by Leeds and York Partnership NHS Foundation Trust.

\section{Monitoring adverse events}

Inherent in the nature of the condition under scrutiny (depression) is the risk of suicide and deliberate selfharm. We will follow good clinical practice in monitoring for suicide risk during all patient encounters with trial participants. Where any risk to young people due to expressed thoughts of self-harm is encountered, these will be notified to the PMHW who will either contact the participant to assess the situation or arrange for an urgent appointment with the daily 'duty clinician' covering urgent calls or the on call psychiatrist (there is a consultant psychiatrist on call at all times). We will also collect session by session outcome measures using the Short Mood and Feelings Questionnaire that includes a question ('I thought about killing myself') which screens for suicidal ideation. Any young person answering this question in the affirmative will be offered an appointment urgently by their key worker (clinician or PMHW) or the trial principal investigator if their key worker is unavailable.

Serious adverse events that are fatal or life-threatening will be recorded and reported to the research ethics committee within 7 days of knowledge of such cases. All other suspected serious unexpected adverse reactions will be reported to the Data Monitoring Ethics Committee (DMEC), Trial Steering Committee (TSC), trial sponsor and ethics committee within 15 days of first knowledge.

\section{Trial Steering Committee}

A TSC will be set up and will include an independent chair and at least two other independent members, along with the lead investigator and the other study collaborators. They will meet three times a year.

\section{Data Monitoring Ethics Committee}

A DMEC will be set up and include an independent chair and at least two independent members. Issues surrounding data collection, ethical issues and any reported serious adverse events will be considered here. The DMEC will meet annually.

Contributors BW was responsible for the overall development of an ethically sound protocol. BW, EL, SG, CV, SB and BA-D were involved in the conception and production of the study and the development of the initial protocol. JA provided methodological expertise while VA provided statistical expertise. LD was the lead researcher on the qualitative component while SA and DT advised on the design and conduct of the health economic analysis. LT assisted with the development and refinement of the protocol during the duration of the trial. All authors made substantial contributions to the drafting, critical revision and final approval of the document.

Funding This article presents independent research funded by the National Institute for Health Research (NIHR) under its Research for Patient Benefit (RfPB) Programme (Grant Reference Number PB-PG-0609-19295).

\section{Competing interests None.}

Patient consent Obtained.

Ethics approval Leeds (West) Research and Ethics Committee (Reference: 10/H1307/137).

Provenance and peer review Not commissioned; internally peer reviewed.

Open Access This is an Open Access article distributed in accordance with the Creative Commons Attribution Non Commercial (CC BY-NC 4.0) license, which permits others to distribute, remix, adapt, build upon this work noncommercially, and license their derivative works on different terms, provided the original work is properly cited and the use is non-commercial. See: http:// creativecommons.org/licenses/by-nc/4.0/

\section{REFERENCES}

1. Papanikolaou K, Richardson C, Pehlivanidis A, et al. Efficacy of anti-depressants in child and adolescent depression: a meta-analytic study. J Neural Transm 2005;113:399-415.

2. National Institute for Health and Clinical Excellence. Depression in children and young people: identification and management in primary, community and secondary care. Clin Guidel 2005;2. http://guidance.nice.org.uk/CG28 (accessed 7 Oct 2011).

3. European Medicines Agency. European Medicines Agency finalises review of anti-depressants in children and adolescents. European Medicines Agency, 2005. http://www.emea.europa.eu/pdfs/human/ press/pr/12891805en.pdf (accessed 7 Oct 2011).

4. European Medicines Agency. European Medicines Agency adopts a positive opinion for the use of Prozac in the treatment of children and adolescents suffering from depression. European Medicines Agency, 2006. http://www.emea.europa.eu/pdfs/human/press/pr/ 20255406en.pdf (accessed 7 Oct 2011).

5. Priest RG, Vize C, Roberts A, et al. Lay people's attitudes to treatment of depression: results of opinion poll for Defeat Depression Campaign just before its launch. BMJ 1996;313:858-9.

6. Grime J, Pollock K. Patients' ambivalence about taking antidepressants: a qualitative study. Pharm J 2003;271:516-19.

7. Goodyer I, Dubicka B, Wilkinson P, et al. Selective serotonin reuptake inhibitors (SSRI) and routine specialist care with and without cognitive behaviour therapy in adolescents with major depression: randomised controlled trial. BMJ 2005;335:142-9.

8. Compton S, March J, Brent D, et al. Cognitive behavioural psychotherapy for anxiety and depressive disorders in children and adolescents. An evidence based medicine review. J Am Acad Child Adolesc Psychiatry 2004;43:930-59.

9. Brent D, Holder D, Kolko D, et al. A clinical psychotherapy trial for adolescent depression comparing cognitive, family and supportive therapy. Arch Gen Psychiatry 1997;54:877-85.

10. Clarke $\mathrm{GN}$, Hornbrook $\mathrm{M}$, Lynch $\mathrm{F}$, et al. A randomised trial of a group cognitive intervention for preventing depression in the offspring of depressed parents. Arch Gen Psychiatry 2001;58:1127-34

11. March J, Silva S, Vitiello B, et al. The treatment for adolescents with depression study (TADS): methods and message at 12 weeks. J Am Acad Child Adolesc Psychiatry 2006;45:1393-403.

12. Harrington R. Depression, suicide and deliberate self-harm in adolescence. Br Med Bull 2001;57:47-60.

13. Proudfoot J, Goldberg DP, Mann A, et al. Computerised, interactive, multimedia cognitive behavioural therapy for anxiety and depression in general practice. Psychol Med 2003;33:217-27.

14. Kaltenthaler E, Brazier J, De Nigris E, et al. Computerised cognitive behaviour therapy for depression and anxiety update: a systematic review and economic evaluation. Health Technol Assess 2006;10:1-186.

15. O'Kearney R, Kang K, Christensen H, et al. A controlled trial of a school based internet program for depressive symptoms in adolescent girls. Depress Anxiety 2009;26:65-72.

16. Abeles P, Verduyn C, Robinson A, et al. Computerised CBT for adolescent depression ("Stressbusters") and its initial evaluation through an extended case series. Behav and Cogn Psychother 2009;37:151-65.

17. Cunningham MJ, Wuthric VM, Rapee RM, et al. The cool teens CD rom for anxiety disorders in adolescents: a pilot case series. Eur Child Adolesc Psychiatry 2009;18:125-9.

18. Spence SH, Holmes JM, March S, et al. The feasibility and outcome of clinic plus internet delivery of cognitive behaviour therapy for childhood anxiety. J Consult Clin Psychol 2006;74:614-21.

19. Burleson Daviss W, Birmaher B, Melhem NA, et al. Criterion validity of the Mood and Feelings Questionnaire for depressive episodes in 
clinic and non-clinic subjects. $J$ Child Psychol Psychiatry 2006;47:927-34.

20. National Institute for Clinical Excellence. Depression: core interventions in the management of depression in primary and secondary care. London: HMSO, 2004.

21. Wood A, Harrington R, Moore A. Controlled trial of a brief cognitive behavioural intervention in adolescent patients with depressive disorders. J Child Psychol Psychiatry 1996;37:737-46.

22. Kuzel A. Sampling in qualitative inquiry. In: Crabtree B, Miller W. eds. Doing qualitative research. Newbury Park, CA: Sage, 1992:31-44.

23. Strauss A, Corbin J. Basics of qualitative research. London: SAGE, 1990
24. Pescosolido B. Illness careers and network ties: a conceptual model of utilization and compliance. Adv Med Sociol 1991;2:161-84.

25. Pescosolido B. Beyond rational choice: the social dynamics of how people seek help. Am J Sociol 1992;97:1096-138.

26. Biddle L, Donovan J, Sharp D, et al. Explaining non-help-seeking amongst young adults with mental distress: a dynamic interpretive model of illness behaviour. Sociol Health IIIn 2007:29:983-1002.

27. Arain $\mathrm{M}$, Campbell MJ, Cooper $\mathrm{CL}$, et al. What is a pilot or feasibility study? A review of current practice and editorial policy. BMC Med Res Methodol, 2010;10:67.

28. Lancaster GA, Dodd S, Williamson PR. Design and analysis of pilot studies: recommendations for good practice. J Eval Clin Pract 2004; 10:307-12. 